The Nepali Math. Sc. Report,Vol. 38, No.2, 2021:13-19

DOI: $0.3126 /$ nmsr.v38i2.42845

\title{
DEGREE OF APPROXIMATION OF SIGNALS BY NÖRLUND SUMMABILITY OF DERIVED FOURIER SERIES
}

\author{
SURESH KUMAR SAHANI ${ }^{1}$ AND LAKSHMI NARAYAN MISHRA ${ }^{2}$ \\ 1 Department of Mathematics, MIT Campus, T.U, Janakpurdham, 45600, Nepal \\ 2 Department of Mathematics, School of Advanced Sciences, Vellore Institute of \\ Technology (VIT) University, Vellore 632014, Tamil Nadu, India \\ Email: sureshkumarsahani35@gmail.com ${ }^{1}$, lakshminarayanmishra04@gmail.com²
}

\begin{abstract}
In this research work, we have introduced generalized Nörlund summability of derived Fourier series and its conjugate series and utilized it to several summability method. Further, a set of new and well known arbitrary result have been obtained by using main theorem. Considering suitable conditions a previous result has been obtained, which validates the current findings.
\end{abstract}

Keywords: Nörlund methods, Fourier series, Summability.

AMS (MOS) Subject Classification.: 40G05,40D05,42A24,etc.

\section{INTRODUCTION}

The hoary, hackneyd and hazy concept of convergence of infinite series was contingent upon robust foundation with the emergence of Cauchy's enduring work course d'Analyses algebraic in 1821 and Abel's researches(see [6]) on the binomial series in 1826. Nonetheless, it was noticed after perusal that certain non- convergent series, specially in Dynamical Astronomy provided almost correct results. In 1890 "a theory of divergent series" was propounded which was pioneering work for the first time. It was at this very time a" paper on the multiplication series ware published by no less a personality than by Cesàro(see [6]). For most of the seminal and pioneering mathematical analyst the theory of series, whose sequence of partial sums fluctuate, has been at the core of creative activity. Through relentless endeavours made by multiple eminent mathematicians that fruitful and satisfactory methods were conceived and concretised towards the fag end of the last century and in the early year of the present century. The method devised was so as to associate them with process closely associated with Cauchy's concept of convergence of which certain values may be called their sums in a reasonable manner. To elaborate and elucidate further. Summability Szász and Hardy(see [6]), the process of associating generalized sum, imparted a natural generalization of classical concept of convergence Hobson, Titchmarsh and is therefore responsible, within the domain applicability, an extensive and former rejected series which used to be forbidden as divergent. In this way, the idea of convergence has been deemed and dubbed as sweeping generalization, for it was needless to say, natural to peruse the possibility of putting forward the concept of convergence. Indeed just as the concept of convergence has

Accepte/Published Online: December, 2021 
resulted in the extension under the general title of summability (Kogbetliantz), the idea of ordinary and absolute convergence have contributed to the development of ordinary and absolute summability in the same way. Thus, the concept of uniform convergence would have definitely underscored the concept uniform summability highlighted prominently by analyst.

Let $\sum a_{n}$ be a given infinite series with the sequence of partial sums. Let $\left\{p_{n}\right\}$ be a sequence of constant, real or complex, and let us write

$$
\begin{aligned}
& P_{n}=p_{0}+p_{1}+p_{2}+\ldots+p_{n}, \\
& \left(P_{-1}=p_{-1}=0\right) \text { and } p_{n} \neq 0 .
\end{aligned}
$$

1.1 Definition (see [1]-[3])

The matrix $P=\left(p_{n k}\right)$, defined by

$$
p_{n k}=\left\{\begin{array}{l}
\frac{p_{n-k}}{p_{n}}, n \geq k \\
0, \quad n<k
\end{array}\right.
$$

is known as the Nörlund matrix means of the sequence $\left\{p_{n}\right\}$.

\subsection{Definition(see [12])}

The FF- transformation

$$
t_{n}=\frac{1}{P_{n}} \sum_{k=0}^{n} p_{n} . s_{n-k} .
$$

by the Nörlund matrix $\mathrm{P}$, defines the sequences $\left\{t_{n}\right\}$, of the Nörlund means $\left(N, p_{n}\right)$ of the sequence $\left\{s_{n}\right\}$. It is well known that the Nörlund matrix is permanent when,

$$
\sum_{k=0}^{n}\left|p_{k}\right|=O\left(\left|P_{n}\right|\right), \text { as } n \rightarrow \infty, \text { then }
$$

and

$$
\lim _{n \rightarrow \infty} \frac{p_{n}}{P_{n}}=0
$$

\subsection{Definition(see [5])}

In case of $p_{n}=\frac{1}{n+1}$, and therefore

$$
P_{n}=\sum_{k=0}^{n} \frac{1}{k+1} \sim \log n \text { as } n \rightarrow \infty, \text { then }
$$

the Nörlund means reduces to harmonic mean and the method is known as harmonic method of summability.

Let $f(t)$ be a continuous function of bounded variation, periodic with the period $2 \pi$ and integrable in the Lebesgue sense in the interval $(-\pi, \pi)$.

Let the function $f(t)$ have a derivative $f^{\prime}(x)$ at $t=x$ and let the Fourier series associated with $f(t)$ be

$$
f(t) \sim \frac{a_{0}}{2}+\sum_{n=1}^{\infty}\left(a_{n} \cos n t+b_{n} \sin n t\right)
$$


DEGREE OF APPROXIMATION OF SIGNALS BY NÖRLUND SUMMABILITY OF DERIVED FOURIER SERIES

where the constants $a_{n}$ and $b_{n}$ are the given by the Euler- Fourier formulae.

The derived series of Fourier series be

$$
\sum_{n=1}^{\infty} n\left(b_{n} \cos n t-a_{n} \sin n t\right)
$$

The series conjugate to $(5)$ is

$$
\sum_{n=1}^{\infty} n\left(a_{n} \cos n t+b_{n} \sin n t\right)
$$

In this paper, we use the following notations:

$$
g(t)=f(x+t)-f(x-t)-2 t \cdot f^{\prime}(x),
$$

and

$$
h(t)=f(x+t)+f(x-t)-2 t \cdot f(x),
$$

Hüseyin Bor ([7]-[10]) studied on the absolute Nörlund summability factors, almost increasing sequences and their new applications, Some new results on infinite series and Fourier series and some equivalence theorems on absolute methods respectively. In 1951, Prasad and Singh [4] have discussed the strong Cesáro Summability of the derived series of a Fourier series and its conjugate series at $t=x$ under the conditions:

$$
\int_{0}^{t}|d g(u)|=O\left[\left\{\frac{t}{\log \frac{1}{t}}\right\}^{1+\epsilon}\right], \epsilon>0
$$

and

$$
\int_{0}^{t}|d h(u)|=O\left[\left\{\frac{t}{\log \frac{1}{t}}\right\}^{1+\epsilon}\right], \epsilon>0 .
$$

\section{Known Theorem}

Tripathi [5] has studied the harmonic summability of these series under the conditions (9) and (10) with $\epsilon=0$. He has proved the following theorem:

\section{Theorem}

The derived series of the Fourier series of a function $f(t)$ is summable by harmonic means to the sum $f^{\prime}(x)$ at the point $t=x$, at which

$$
\int_{0}^{t}|d g(u)|=O\left(\frac{t}{\log \frac{1}{t}}\right) .
$$

In the present paper, the above theorem has been generalized by replacing the harmonic summability by a more general Nörlund summability. We establish the following theorem on the Nörlund summability of the derived series of Fourier series and its allied series.

\section{Main Theorem}

Theorem: If $\left\{p_{n}\right\}$ is a non-negative, non-increasing sequences of constants such that

$$
\sum_{k=2}^{n}\left(\frac{p_{k}}{k \log k}\right)=O\left(P_{n}\right)
$$


then the series (5) is summability by Nörlund method $\left(N, p_{n}\right)$ to the sum $f^{\prime}(x)$ at the point $t=x$ which the condition

$$
G(t)=\int_{0}^{t}|d g(u)| O\left[\frac{t}{\log \frac{1}{t}}\right] \text { is satishfied. }
$$

The following Lemma is required for the proof of the theorem.

Lemma (see[11]):

If $\left\{p_{n}\right\}$ is a non-negative, non-increasing sequences of constants then for $0 \leq a \leq b \leq \infty, 0<$ $t \leq \pi$ and any $\mathrm{n}$,

$$
\left[\sum_{k=0}^{b} p_{k} \frac{\sin \left(n-k+\frac{1}{2}\right) t}{\sin \frac{t}{2}}\right]=O\left[\frac{P\left(\frac{1}{t}\right)}{t}\right] .
$$

Denoting by $\sigma_{n}(x)$ to the sum of first $\mathrm{n}$ terms of the series (5) at point $t=x$, we get

$$
\begin{gathered}
\sigma_{n}(x)=\frac{1}{2 \pi} \int_{0}^{2 \pi}\left\{\frac{d}{d x}\left(\frac{\sin \left(n+\frac{1}{2}\right)(x-t)}{\sin \frac{x-t}{2}}\right)\right\} f(t) d t \\
=-\frac{1}{2 \pi} \int_{0}^{2 \pi} f(t)\left\{\frac{d}{d x}\left(\frac{\sin \left(n+\frac{1}{2}\right)(x-t)}{\sin \frac{x-t}{2}}\right)\right\} d t \\
=-\frac{1}{2 \pi} \int_{0}^{2 \pi}\{f(x+t)-f(x-t)\} \frac{d}{d t}\left(\frac{\sin \left(n+\frac{1}{2}\right) t}{\sin \frac{t}{2}}\right) d t
\end{gathered}
$$

Integrating by parts, we get

$$
\sigma_{n}(x)=\frac{1}{2 \pi} \int_{0}^{\pi} \frac{\sin \left(n+\frac{1}{2}\right) t}{\sin \frac{t}{2}} d g(t)+f^{\prime}(x)
$$

Hence,

$$
\sigma_{n}(x)-f^{\prime}(x)=\frac{1}{2 \pi} \int_{0}^{2 \pi} \frac{\sin \left(n+\frac{1}{2}\right) t}{\sin \frac{t}{2}} d g(t) .
$$

or

$$
\sigma_{n-k}(x)-f^{\prime}(x)=\frac{1}{2 \pi} \int_{0}^{\pi} \frac{\sin \left(n-k+\frac{1}{2}\right) t}{\sin \frac{t}{2}} d g(t) .
$$

Concerning the FF transformation by Nörlund matrix

$$
P=\left(p_{n k}\right) \text { of the sequence }\left\{\sigma_{n-k}(x)-f^{\prime}(x)\right\}
$$

we have

$$
\begin{gathered}
\frac{1}{P_{n}} \sum_{k=0}^{n} p_{k}\left(\sigma_{n-k}(x)-f^{\prime}(x)\right)=\frac{1}{P_{n}} \sum_{k=0}^{n} p_{k} \frac{1}{2 \pi} \int_{0}^{\pi} \frac{\sin \left(n+\frac{1}{2}\right) t}{\sin \frac{t}{2}} d g(t) \\
=\int_{0}^{\pi} d g(t) \frac{1}{2 \pi P_{n}} \sum_{k=0}^{n} p_{k} \cdot \frac{\sin \left(n-k+\frac{1}{2}\right) t}{\sin \frac{t}{2}} \\
=\int_{0}^{\pi} N_{n}(t) d g(t)
\end{gathered}
$$


DEGREE OF APPROXIMATION OF SIGNALS BY NÖRLUND SUMMABILITY OF DERIVED FOURIER SERIE\$ where

$$
N_{n}(t)=\frac{1}{2 \pi P_{n}} \sum_{k=0}^{n} p_{k} \cdot \frac{\sin \left(n-k+\frac{1}{2}\right) t}{\sin \frac{t}{2}} .
$$

Thus, in order to prove the theorem what is to be chosen is that

$$
\int_{0}^{\pi} N_{n}(t) d g(t)=O(1) \text {, as } n \rightarrow \infty .
$$

Let us write

$$
\begin{gathered}
\int_{0}^{\pi} N_{n}(t) d g(t)=\left(\int_{0}^{\frac{1}{n}}+\int_{\frac{1}{n}}^{\delta}+\int_{\delta}^{\pi}\right) N_{n}(t) d g(t), \text { where } 0<\delta<\pi \\
=M_{1}+M_{2}+M_{3}(\text { say }), \quad 0<\frac{1}{n}<\delta \leq \pi
\end{gathered}
$$

First Consider,

$$
\begin{gathered}
M_{1}=\int_{0}^{\frac{1}{n}} N_{n}(t) d g(t) \\
=O\left[\frac{1}{P_{n}} \int_{0}^{\frac{1}{n}} \sum_{k=0}^{n} p_{k} \frac{\left|\sin \left(n-k+\frac{1}{2}\right) t\right|}{\left|\sin \frac{t}{2}\right|}|d g(t)|\right. \\
=O\left|\frac{1}{P_{n}} \int_{0}^{\frac{1}{n}} \sum_{k=0}^{n} n p_{n}\right| d g(t)|| \\
=O\left[\int_{0}^{\frac{1}{n}}|d g(t)|\right] \\
=O(1) .
\end{gathered}
$$

Next, by virtue of the Riemann-Lebesgure theorem and because Nörlund matrix is permanent, we have

$$
M_{3}=\int_{0}^{\pi} N_{n}(t) d g(t)=O(1) \text {, as } n \rightarrow \infty .
$$

Again, applying the lemma with $\mathrm{a}=0, \mathrm{~b}=\mathrm{n}$ in (16) we have

$$
\begin{aligned}
\left|N_{n}(t)\right| & =O\left(\frac{1}{P_{n}} \cdot \frac{P_{\tau}}{t}\right) \\
& =O(1)
\end{aligned}
$$

where $\tau=\left[\frac{1}{t}\right]$.

Hence,

$$
M_{2}=\int_{\frac{1}{n}}^{\delta} N_{n}(t) d g(t)=O\left(\int_{\frac{1}{n}}^{\delta}\left|N_{n}(t)\right||d g(t)|\right)
$$




$$
\begin{gathered}
=O\left(\frac{1}{p_{n}} \int_{\frac{1}{n}}^{\delta} \frac{p_{\tau}}{t}|d g(t)|\right) \\
=O\left(\frac{1}{p_{n}}\left\{\left[G(t) \frac{p_{\tau}}{t}\right]_{\frac{1}{n}}^{\delta}-\int_{\frac{1}{n}}^{\delta} G(t) d\left(\frac{p_{\tau}}{t}\right)\right\}\right) \\
=O(1)+O\left(\frac{1}{p_{n}} \int_{\frac{1}{n}}^{\delta} \frac{t}{\log \left(\frac{1}{n}\right)} d\left(\frac{p_{\tau}}{t}\right)\right) .
\end{gathered}
$$

Now,

$$
\begin{aligned}
& \int_{\frac{1}{n}}^{\delta} \frac{t}{\log \left(\frac{1}{t}\right)} d\left(\frac{p_{\tau}}{t}\right)=O\left(\int_{\frac{1}{\delta}}^{n} \frac{1}{x \log x} d\left(x p_{[x]}\right)\right) \\
= & O\left(\int_{\frac{1}{\delta}}^{\alpha} \frac{1}{x \log x} d\left(x \cdot p_{[x]}\right)+\int_{\alpha}^{n} \frac{1}{x \log x} d\left(x \cdot p_{[x]}\right)\right) \\
= & O(1)+O\left(\int_{\alpha}^{n} \frac{1}{x \log x} d\left(x \cdot p_{[x]}\right)\right) \\
= & O(1)+O\left(\sum_{k=\alpha}^{n} \frac{\Delta\left(k \cdot p_{k}\right)}{k \cdot \log k}\right) \\
= & O(1)+O\left(\sum_{k=\alpha}^{n}\left\{\frac{(k+1) \cdot p_{k+1}}{k \cdot \log k}-\frac{k \cdot p_{k}}{k \cdot \log k}\right\}\right) \\
= & O\left(P_{n}\right) \cdot \\
= & O(1)+O(1)+O\left(\sum_{k=\alpha}^{n}\left\{\frac{\left(p_{k+1}-p_{k}\right)}{\log k}-\frac{p_{k+1}}{k \cdot \log k}\right\}\right) \\
= & \left(\sum_{k=\alpha}^{n} \frac{p_{k+1}}{\log k}\right) \\
& \\
& \\
&
\end{aligned}
$$

Hence, (22) and (23) gives

$$
L_{2}=O(1)
$$

Thus, (19), (20) and (24) prove (18)and hence, the result follows from (15). 


\section{Conclusions}

The objective of the paper is to get least conditions for Nörlund summability of derived Fourier series. The idea of summability of infinite series has been applied in almost all application areas of science like rectifications of signals, orthogonal series, to speed up the rate of convergence and approximations theory etc. Also, BIBO stability has been enhanced by determining least set of sufficient condition for Nörlund summability. Using these techniques, the output of the waves can be made more balanced and to analyse the behavior of input. By the examination, we may find that our hypothesis is a summed up variant which can be diminished for a few notable summabilities as appeared in corollaries.

\section{REFERENCES}

[1] H. Bor, A note on absolute Nörlund summability, Journal of Inequalities in Pure and Applied Mathematics, Vol. 6, Issue 4, Article115, pp 1-5,2005.

[2] V.N. Mishra, K. Khatri, and L. M. Mishra, Application of functions belonging to $\operatorname{Lip}\{\sigma(t), r\}$ class by $\left(N, p_{n}\right)(E, q)$ Summability of Congugate series of Fourier series, Journal of Inequatility and applications, pp. 1 - 10,2012.

[3] X.Z. Krasniqui, On approximation of functions belonging to some classes of functions by $\left(N, p_{n}, q_{n}\right)(E, Q)$ means of conjugate series of its Fourier series, Khayyam J. Math.Vol. 6 No.1, pp.73 86,2020 .

[4] B.N. Prasad and V. N. Singh, On the strong summability of the derived Fourier Series and its Conjugate series Math Zeit,Vol. 56 , pp.280 - 288, 1951.

[5] L.M. Tripathi, On the harmonic summability of the derived Fourier series and its conjugate series. Proc. Nat. Acad. sci. India, sec. A,Vol. 33 , pp.443 - 454, 1963.

[6] H.K. Nigam, Birth and growth of summability and approximation theory, International Journal of Pure and Applied Mathematics, Vol. 83, No. 5, 639-641, 2013.

[7] H. Bor, On the absolute Nörlund summability factors, Mathematical communications, Vol. 5 , pp.143 147,2000 .

[8] H. Bor, Almost increasing sequences and their new applications, Journal of Inequalities and Applications,Vol. 1, pp. 207 - 213, 2013.

[9] H. Bor, Some new results on infinite series and Fourier series, Positivity, pp.467 - 473, 2015.

[10] H. Bor, Some equivalence theorems on absolute methods, Acta Mathematica Hungarica,Vol. 1, pp.208 - 214, 2016.

[11] T. Singh on the Nörlund summability of Fourier series and its Conjugate series, Proc. Nat. Insiti. of Sci. of India, Vol.29(A), pp.65-73,1963.

[12] S. Lal and V.N. Tripathi, On the study of derived series of a Fourier series and allied series by almost Nörlund Summability method, Acta Ciencia Indica,Vol. XXVIIIM, No. 1, 77-88, 2002. 